\title{
Accurate and Complete Hardware Profiling for OpenMP
}

\section{Document Version}

Accepted author manuscript

Link to publication record in Manchester Research Explorer

\section{Citation for published version (APA):}

Neill, R., Drebes, A., \& Pop, A. (Accepted/In press). Accurate and Complete Hardware Profiling for OpenMP. In Proceedings of the 13th International Workshop on OpenMP: Scaling OpenMP for Exascale Performance and Portability (Lecture Notes in Computer Science (LNCS)). Springer Nature.

\section{Published in:}

Proceedings of the 13th International Workshop on OpenMP

\section{Citing this paper}

Please note that where the full-text provided on Manchester Research Explorer is the Author Accepted Manuscript or Proof version this may differ from the final Published version. If citing, it is advised that you check and use the publisher's definitive version.

\section{General rights}

Copyright and moral rights for the publications made accessible in the Research Explorer are retained by the authors and/or other copyright owners and it is a condition of accessing publications that users recognise and abide by the legal requirements associated with these rights.

\section{Takedown policy}

If you believe that this document breaches copyright please refer to the University of Manchester's Takedown Procedures [http://man.ac.uk/04Y6Bo] or contact uml.scholarlycommunications@manchester.ac.uk providing relevant details, so we can investigate your claim.

\section{OPEN ACCESS}




\title{
Accurate and Complete Hardware Profiling for OpenMP
}

\section{Multiplexing Hardware Events Across Executions}

\author{
Richard Neill, Andi Drebes, and Antoniu Pop \\ School of Computer Science, The University of Manchester, Manchester, UK \\ first. last @manchester.ac.uk
}

\begin{abstract}
Analyzing the behavior of OpenMP programs and their interaction with the hardware is essential for locating performance bottlenecks and identifying performance optimization opportunities. However, current architectures only provide a small number of dedicated registers to quantify hardware events, which strongly limits the scope of performance analyses. Hardware event multiplexing can help cover more events, but incurs a significant loss of accuracy and introduces overheads that change the behavior of program execution significantly. In this paper, we present an implementation of our technique for building a unique, coherent profile that contains all available hardware events from multiple executions of the same OpenMP program, each monitoring only a subset of the available hardware events. Reconciliation of the execution profiles relies on a new labeling scheme for OpenMP that uniquely identifies each dynamic unit of work across executions under dynamic scheduling across processing units. We show that our approach yields significantly better accuracy and lower monitoring overhead per execution than hardware event multiplexing.
\end{abstract}

Keywords: Performance analysis, hardware events, performance monitoring counters, OpenMP profiling

\section{Introduction}

Monitoring hardware behavior during the execution of an OpenMP program can reveal performance bottlenecks arising from the parallel structure of the software, characteristics of the hardware, and the complex interactions over shared resources. This behavior can be investigated by counting the number of occurrences of specific hardware events during execution using Performance Monitoring Counters (PMCs; dedicated on-chip registers). The counts can then be related to the individual regions of an OpenMP program during post-mortem performance analysis to enable reasoning about the variation in performance and hardware behavior across the program's execution. Various tools use hardware event monitoring to enable performance analysis of parallel programs $[4,19,2,1]$. However, as the number of available PMCs is generally orders of magnitude lower than the number of hardware events, only a small fraction of available hardware 
events can be monitored simultaneously, which severely limits analyses involving multiple hardware events. Furthermore, there are architecture-specific design constraints for PMCs that often result in incompatibilities between events. Certain sets of events can therefore not be monitored simultaneously with other events, even if there are enough PMCs.

Hardware Event Multiplexing (HEM) is a common technique for alleviating the limitations imposed by event incompatibility and a lack of PMC availability. It consists of time-sharing the PMCs: subsets of hardware events are regularly rotated to be monitored, intending that each event is monitored over a representative sample of the execution. The Linux kernel's perf_event subsystem provides a standard implementation of HEM, although other works have attempted to enhance HEM by improving its interpolation mechanism between sampled counts $[9,10,8]$ or optimizing its event scheduler to achieve better coverage of events [3]. However, because HEM time-shares the events, for any particular profiled event, there are necessarily periods of execution during which the event is not monitored. In previous work [14], we investigated the accuracy of profile data acquired from OpenStream [17] programs via HEM, and found that HEM is unable to produce accurate hardware event values for dynamic fine-grained task-parallel programs. In [14] we presented a significantly more accurate profiling technique for OpenStream programs, where data acquired during multiple executions is fused into a single, coherent execution profile. A somewhat related concept, known as trace alignment $[6,12]$, was studied for sequential programs, but it hinges on identifying sequentially ordered execution phases, and is therefore not applicable to OpenMP's dynamic parallel schedules. To our knowledge, there is no other work which aims to reconcile the hardware event performance data observed in distinct executions to produce a complete, consistent view of hardware behavior of OpenMP programs.

In this paper, we present a multi-execution combination approach for OpenMP programs and evaluate its accuracy in comparison to HEM. Our approach targets a model-centric analysis and therefore focuses on monitoring at the granularity of execution units (XUs), a term we use in this paper to refer to two kinds of dynamically executed work in an OpenMP program: an executed instance of an OpenMP task construct or an executed range of iterations from an OpenMP for loop instance. Accurate performance monitoring data at this granularity is important to allow for a statistical approach to performance analyses, enabling the detection of performance bottlenecks arising from program and architecture characteristics, such as in $[5,11]$. Furthermore, by ensuring performance data reliability at this granularity, we enable all analyses that operate at higher levels of abstraction, ensuring, for example, accurate measurements of performance metrics aggregated across sets of XUs, e.g., per core, per NUMA node or per OpenMP construct.

This paper makes the following two main contributions:

- We present a labeling scheme for OpenMP XUs, which uniquely identifies each XU irrespective of runtime scheduling, ensuring that XUs are identifiable across multiple dynamic executions. 
- We present an implementation of our multi-execution combination approach for OpenMP and evaluate its accuracy against HEM. We implement three strategies for combination: Label Graph Location, Behavior Clustering, and present a new strategy called Chronological Type Combination.

We describe our use of the Aftermath-OpenMP [4] instrumented OpenMP runtime to generate per-XU hardware event monitoring data in Section 2. An overview of our multi-execution combination approach and implementation strategies are detailed in Section 3. Section 4 presents our new OpenMP labeling scheme. Section 5 contains our evaluation for the combination approaches and HEM.

\section{Generating OpenMP Execution Profiles}

Our approach operates on the set of all $X U s$ executed during a program and their associated performance monitoring data. Our definition of XUs is based on the higher-level OpenMP language constructs rather than on their generated code and dynamic execution. This means that even if the runtime system reaches a scheduling point and breaks down an XU into multiple chunks, possibly executing across multiple worker threads, the $\mathrm{XU}$ is considered to be the sum of its parts.

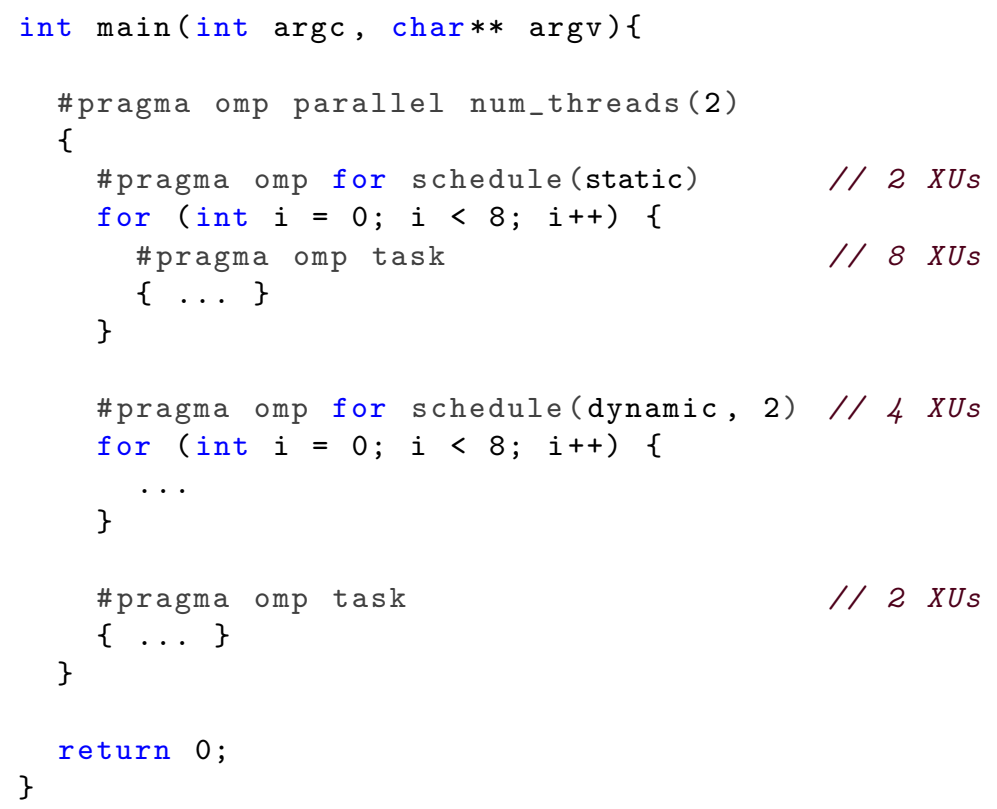

Listing 1.1. Example OpenMP program and resulting execution units. 
The example OpenMP program in Listing 1.1 creates 16 XUs as follows. On Line 5, the static schedule of the for construct implies that each of the two worker threads declared in the team of the parallel construct (Line 3) receives half of the iterations, which makes up 2 XUs. Each iteration of this same loop further creates one task on Line 7, resulting in 8 XUs. In the second for construct, in Line 11, the dynamic schedule with a chunk size of 2 results in 4 XUs, each containing 2 iterations. Finally, the last task construct, on Line 16, occurs within the body of the parallel region, so each of the two worker threads will create their own instance, creating the last 2 XUs.

A set of hardware events are monitored during a profiling execution of a program. Each executed XU $u$ is therefore associated with a set of pairs composed of the event and the number of occurences during the execution of $u$, referred to as the event counts. In this paper, we define the function count_set $(u, E)$ to return the set of counts associated to $u$ for all events in the event set $E$, where for simplicity $E$ is the complete set of hardware events associated with $u$ if the second argument of the function is omitted. For a given execution, we refer to the entire set of XUs together with their complete set of profiled event counts as an execution profile, or profile for short. We finally define the type of an XU to be a syntactic identifier for its corresponding language construct within the code, with $\mathcal{T}$ being the set of all XU types defined in the program specification.

We rely on Aftermath-OpenMP [4] to trace OpenMP execution and enable generation of execution profiles. In this paper, we note $\mathcal{E}$ to be the set of available hardware events on the system. As described in the introduction, hardware event monitoring is limited by the available PMCs. Assuming for simplicity that there are no incompatibilities and any hardware event can be monitored in any PMC, event counts cannot be associated with all XUs in an Aftermath-OpenMP execution profile when the number of events to be profiled exceeds the number of available PMCs $N_{\mathrm{PMC}}$. To overcome this problem, we combine the data of XUs profiled during multiple distinct executions into a single, coherent profile where all XUs are associated with event counts for each event in $\mathcal{E}$, thus achieving complete execution profiles.

\section{Execution Profile Combination}

To generate a complete execution profile, where each XU has hardware event values for all available hardware events, our approach is to execute a program repeatedly and to monitor a different set of hardware events for each run, instead of rotating hardware events over the duration of a single execution as in HEM. The different profiles are then combined using one of the three strategies described below. Among them, the Chronological Type Combination is new in this paper, while the other two have been adapted to OpenMP from our previous work [14], notably requiring the development of an XU labeling scheme.

Execution profile combination starts by generating $n$ subsets of $\mathcal{E}$, where each subset can be monitored simultaneously on the system. The union of all subsets must cover $\mathcal{E}$, but subsets need not be exclusive and event overlaps can even be 


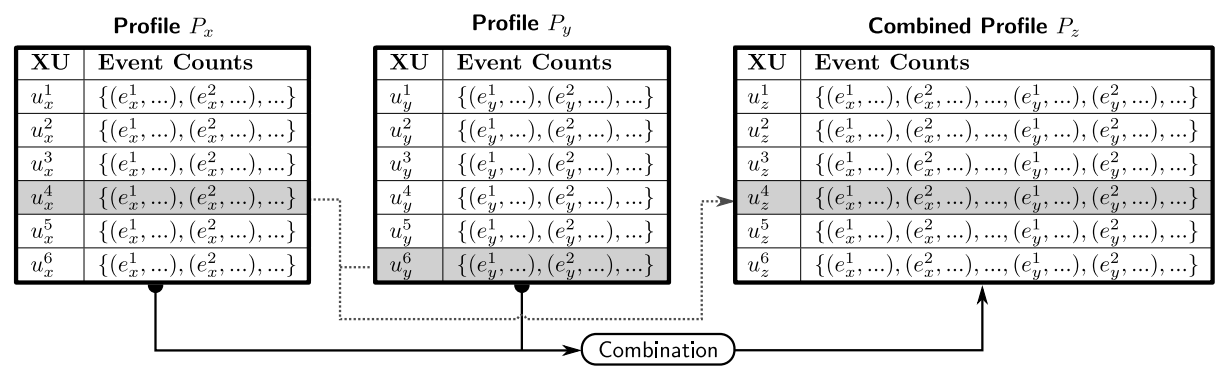

Fig. 1. Combination of two profiles. The highlights denote an example derived XU $u_{z}^{4}$ that is generated from two source XUs $u_{x}^{4}$ and $u_{y}^{6}$.

necessary depending on the combination strategy. The target program is then executed $n$ times with the same input and configuration but with each execution monitoring a different event subset, to produce a set of execution profiles.

The goal of execution profile combination is to apply a combination to these profiles in order to produce a combined profile. A combined profile consists of a set of derived XUs that are each generated from a unique set of XUs $\left\{u_{1}, \ldots, u_{n}\right\}$, with each XU's subscript indicating a different source execution profile. A derived $\mathrm{XU}$ is therefore associated with the hardware event monitoring data from each of the associated XUs. This ensures that it has monitored hardware event counts for all events in $\mathcal{E}$, where each associated event count was observed in an execution during which the event was monitored throughout the entire duration. Figure 1 illustrates the approach in the case of a combination of two profiles $P_{x}$ and $P_{y}$, where for clarity we include a superscript identifier with each XU. The highlighted XUs and the dotted line represent an example of a combination step, in which the task profiles of $u_{x}^{4}$ and $u_{y}^{6}$ are combined for $u_{z}^{4}$.

Selecting the set of XUs to combine across distinct executions of an OpenMP program is non-trivial. OpenMP programs can exhibit highly dynamic behavior at execution time, such that two executions of the same program with identical input may vary significantly. Such variability is unavoidable in modern systems. Firstly, because of the complexity of the hardware, possibly starting execution in different states (e.g., CPU power-modes), the complex interactions between the software and hardware (e.g., the cache hierarchy, memory prefetcher, branch predictor), or interference from the software stack (e.g., the OS scheduler). Secondly, because the execution of OpenMP constructs (e.g., task, for) can be scheduled dynamically to worker threads by the runtime system. Furthermore, as the same workers may be assigned entirely different work during different executions, particularly for OpenMP programs that specify very unbalanced work shares through the use of single, master or section directives, this may cause partial reordering of work between different executions. If the behavior at execution time of selected XUs significantly varied between the executions, then the combination may produce inaccurate profiles that have hardware event counts that are not representative of what occurs in any single program execution. To alleviate these issues, we have devised the following combination strategies. 
Chronological Type Combination Assuming that executions could be identical in their scheduling, system environment and behavior at execution time, then the execution times and allocation of XUs to worker threads across distinct executions of the program would be the same. Moreover, one would expect the same hardware events to occur at the same relative time across each execution of the program. Under these optimistic assumptions, a combination strategy that selects XUs of the same type by the chronological order of their start timestamps, called Chronological Type Combination (CTC), is a natural first choice.

Let $\left\{P_{1}, \ldots, P_{n}\right\}$ be the unordered set of execution profiles to be combined via CTC into a resulting combined profile $P_{\mathrm{CTC}}$. Let further $\left\{U_{1}, \ldots, U_{n}\right\}$ denote the corresponding sets of XUs within each profile. Here we assume the basic case, where events monitored during an execution are not also monitored during any other execution. If the event sets of executions overlap, then the first profile containing each event contributes the event's counts to the combined profile, and subsequent monitored event values are unused and therefore redundant. The CTC combination strategy is as follows:

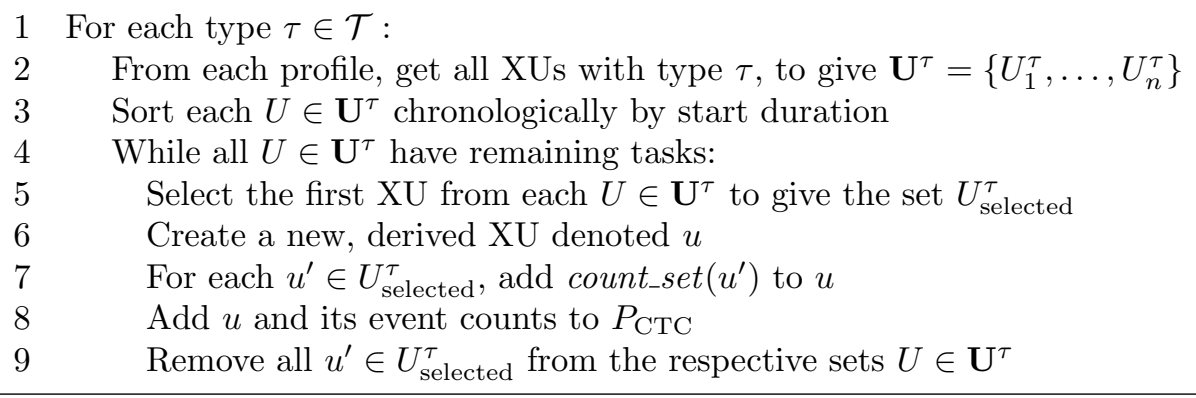

Label Graph Location The Label Graph Location (LGL) combination strategy maps the XUs from multiple profiles by uniquely identifying each dynamic instance across executions. These XUs are of the same type, have identical input data and generate identical output data, and are created following the same path of execution through OpenMP constructs in the code. In order to combine OpenMP programs with LGL, we attach to each XU a unique identifier called a label, that is consistent for that XU across dynamic executions. We define a function label that associates a label to an XU, which we present for OpenMP programs in Section 4. The LGL combination strategy selects those XUs with matching labels across executions.

The LGL combination, as with CTC, operates on an unordered set of profiles $\left\{P_{1}, \ldots, P_{n}\right\}$, again assuming no overlapping events between the monitored executions. The combined profile $P_{\mathrm{LGL}}$ is generated as follows:

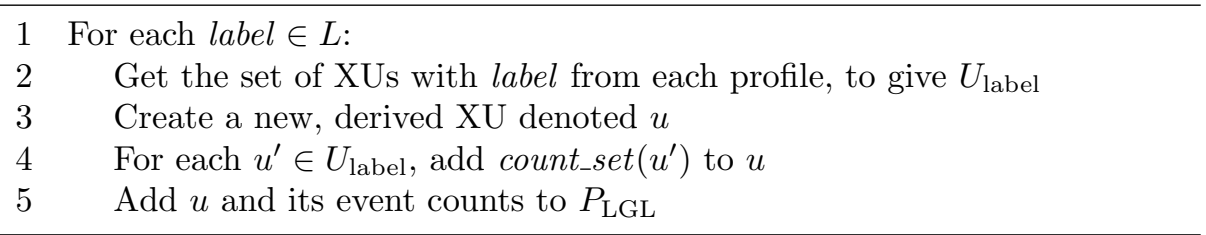


Behavior Clustering The Behavior Clustering (BC) combination strategy accounts for dynamic execution variation between profiles by selecting XUs of the same type that had similar hardware behavior at execution time in each profile. This means that XUs that are combined by BC may be different sets of iterations of the same parallel loop, or different instances of the same task construct, but that exhibit more similar hardware behavior (e.g., interaction with cache) than XUs with equivalent label across executions.

$\mathrm{BC}$ combination operates on an ordered set of profiles $\left\langle P_{1}, \ldots, P_{n}\right\rangle$ with corresponding sets of events monitored during each execution $\left\langle E_{1}, \ldots, E_{n}\right\rangle$. The $\mathrm{BC}$ process is recursive, combining two profiles in each recursion step to accretively build up to the final combined profile. At each step, the resulting profile of the previous combination $P_{\text {prev }}$ is combined with the next profile in the ordered set $P_{\text {cur }}$ to produce the profile $P_{\text {combined }}$, which becomes $P_{\text {prev }}$ for the next step. The first step combines $P_{1}$ and $P_{2}$ directly, with the final profile therefore produced after $n-1$ combinations.

$\mathrm{BC}$ combines two profiles by selecting the XUs that behaved similarly during each execution, with respect to a subset of hardware events. To do this, in constrast to CTC or LGL, BC requires overlapping events between $P_{\text {prev }}$ and $P_{\text {cur }}$, such that $E_{\text {prev }} \cap E_{\text {cur }} \neq \emptyset$. The process is defined as follows:

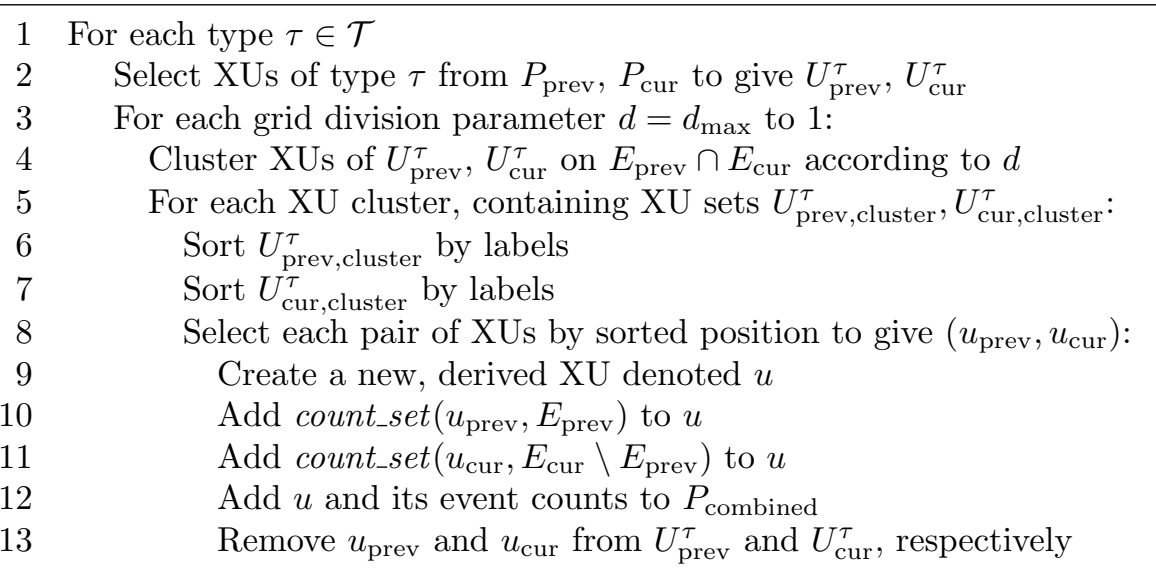

In line $4, \mathrm{XUs}$ are clustered. To do this, a grid is defined of dimension $N_{\mathrm{dim}}$, where each dimension corresponds to an overlapping hardware event. The value range for each event is divided by a grid division parameter $d$ to produce $d^{N_{\text {dim }}}$ cells, which we term clusters. The XUs from each profile are then allocated to clusters according to their values for each of the overlapping events, where in Line 8, XUs are selected for combination by corresponding position in the labelordered sets. As there may not be the same number of XUs from $P_{\text {prev }}$ and $P_{\text {cur }}$ populated in each cluster, not all XUs may be combined after this clustering process. To ensure that all XUs can be combined, the clustering process is repeated with a decremented grid division parameter, resulting in larger clusters and a reduced constraint on the event count similarity of selected XUs. This process continues until at least one profile is exhausted of XUs. 
In this paper, we also evaluate a slightly modified version of $\mathrm{BC}$, where the combined XU pairs within each cluster are selected at random and not according to the label order. This was done to evaluate whether labeling is necessary for $\mathrm{BC}$, and therefore BC's applicability to OpenMP runtimes which do not carry out the necessary instrumentation to enable labeling of XUs.

All three combination strategies are designed to combine execution profiles of the same program with the same inputs, differing in their handling of XU behavior variation between profiles. The three strategies may also be applied to non-deterministic programs with different numbers of XUs across the executions. In this case, the CTC and BC combined profiles consist of the maximum number of XUs that exist across all execution profiles, for each task type. The LGL combined profile consists of XUs corresponding to the subset of labels present in all profiles.

The LGL and BC combination strategies are described in further detail in [14]. To work on OpenMP programs, LGL and BC require the labeling scheme presented in the next section.

\section{Labeling OpenMP Programs}

Both LGL and BC rely on a labeling scheme that uniquely identifies the same $\mathrm{XU}$, as statically defined in the parallel structure of the program, across a set of executions. The labeling is independent from any external influence, such as the scheduling of XUs or resource allocation. The notion of identity is similar for the identity that we defined for task-parallel languages in [14]. In such models, each task $t$ except the root task, representing the initial sequential execution of the program, has a parent task $t_{p}$ that is defined as the task that executed the instructions creating $t$. Two tasks $t$ and $t^{\prime}$ are considered identical iff their respective parents are identical and iff the number of tasks created by their respective parents $t_{p}$ and $t_{p}^{\prime}$ before $t$ and $t^{\prime}$ is the same. The label of a task is defined as the concatenation of the label of its parent, a delimiter and the number of tasks created by its parent before its own creation with an artificial label ' 0 ' for the root task. Since the execution within a task is sequential, the labels are independent of the actual order of task execution by the scheduler and allows tasks to be identified reliably across executions.

We now extend this scheme to the XUs of OpenMP programs.

\subsection{Labeling Scheme}

An XU can only be created if the creating thread has previously encountered one or more OpenMP constructs, as an OpenMP task or iteration range must be specified within at least one parallel construct or for construct, respectively. As OpenMP supports nested parallelism, XUs may be created during the execution of others. For the remainder of this section, we define the function par to return the parent XU or OpenMP construct of another XU or OpenMP construct. To define the labeling scheme, we make a distinction between an XU and 
an OpenMP construct. A construct is the entity in the structure of an OpenMP program corresponding to an omp pragma that a worker can encounter during its execution, whereas an XU is an executable instance of a task construct or block of iterations from a for construct. For example, referring to the program given in Listing 1.1, the OpenMP runtime creates the first iteration range XU spanning four iterations after the thread encounters a parallel construct and a subsequent for construct. The labeling function label applies to an XU when it is initially created, or to an instance of a worker encountering a construct, and we refer to either as $u c$. A worker encountering a task construct and creating a task instance $\mathrm{XU}$ are equivalent with respect to the labeling, and are treated as the same $u c$.

The labeling function is defined as follows:

$$
\text { label }(u c)= \begin{cases}\text { ' } 0 \text { ' } & \text { if } u c \text { is root } \\ \operatorname{label}(\operatorname{par}(u c)) \oplus \operatorname{sibidx}(u c) & \text { otherwise }\end{cases}
$$

Where $\oplus$ denotes the concatenation of two labels with a delimiter. The function sibidx returns a value depending on the kind of $u c$ :

$\operatorname{sibidx}(u)= \begin{cases}\text { start_iteration }(u c) & \text { if } u c \text { is iteration range } \\ \text { next_rank }(\operatorname{par}(u c)) \oplus \operatorname{addr}(u c) & \text { if } u c \text { is for } \\ n e x t \_r a n k_{-} T I D(u c) \oplus a d d r(u c) & \text { if } u c \text { in task, parallel, section }\end{cases}$

In this rule set, start_iteration $(u c)$ returns the index of first executed iteration of the iteration set $u c$ as the label index, and $a d d r(u c)$ returns the memory address of the source location of the OpenMP construct associated to $u c$. A rank is a monotonically increasing integer that is initialized to 1 and represents the rank of $u c$ in the creation order of it and its siblings. The function next_rank $(\operatorname{par}(u c))$ therefore retrieves the next sibling index from the parent. This means that the label indexes are dependent on an order existing between $u c$ and its siblings. As it is legal for a task, section or parallel construct to be defined directly inside a parent parallel construct, the semantics of the program define no order that they are encountered by the threads in the parallel team. In this special case, $u c$ 's rank within its parent parallel construct is set to 0 , and a further label index is concatenated using the TID that created the instance of $u c$ with respect to the number of workers in the team. This is a forced ordering that ensures unique labels for all XUs are maintained. The function next_rank_TID carries out this special case of the labeling scheme, should it occur:

$$
\begin{aligned}
& \text { next_rank_TID }(u c)=
\end{aligned}
$$

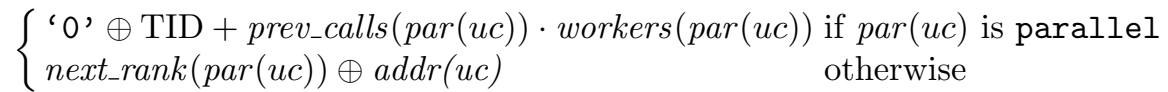

A for construct can also occur directly within a parent parallel construct. However, the XUs created from it will be strictly ordered by their start iteration so do not require special labeling.

In the presented labeling scheme, single, master and critical constructs were not included. This is because they are not necessary for the purposes of 
labeling XUs that are encountered within them, as they either define which worker should create the XU or enforce timing constraints on the creation, both of which the labeling scheme is insensitive to.

For the experimental evaluation, we have implemented the labeling scheme into Aftermath-OpenMP, such that profiling data from different executions can be related to the same instances of work as defined in the program code, subject to the aforementioned variability at execution time.

By labeling the XUs of an OpenMP program, the set of XUs and their creation relationships can be depicted as a Label Graph. Each node of this graph represents an XU or OpenMP construct and edges represent the casual relationships between their creations in the program.

\subsection{Labeling Example}

Figure 2 shows the label graph for the example OpenMP program in Listing 1.1 with 16 profiled XUs. The label for each of the XUs highlighted in gray can be constructed by concatenating the edge indexes and OpenMP construct line numbers, descending the graph in order from root to the XU. In this example, the line numbers represent the memory locations given as the result of addr in the above ruleset, and the oval-shaped entities are included only to aid clarity and do not represent actual OpenMP constructs. The diamond-shaped edge indexes represent forced ranking, where there is no strict ordering for these XUs with respect to their siblings in the semantics of the OpenMP program.

In the next section, we use the labels together with our combination strategies to evaluate the capability of our approach to produce full and accurate hardware event monitoring data for OpenMP programs.

\section{Evaluation}

We evaluate our approach with the different combination strategies using $E x$ ecution Profile Dissimilarity (EPD), a metric derived from the Earth Mover's Distance (EMD) [18]. EPD quantifies the relative accuracy of generated performance monitoring data, where we define accuracy as its similarity with performance monitoring data produced in reference executions. In a reference execution, all of the hardware events are monitored simultaneously, i.e., without any form of multiplexing or combination. A lower EPD therefore represents higher accuracy and thus better reliability when used for analysis.

In this section we first briefly outline EMD before describing its usage for EPD as presented in earlier work [14]. Following this, we describe the experimental setup and present our results.

\subsection{The Earth Mover's Distance}

The EMD, as presented in [18], calculates the similarity between two histograms, here denoted $p$ and $q$. EMD conceptually calculates the minimum amount of 


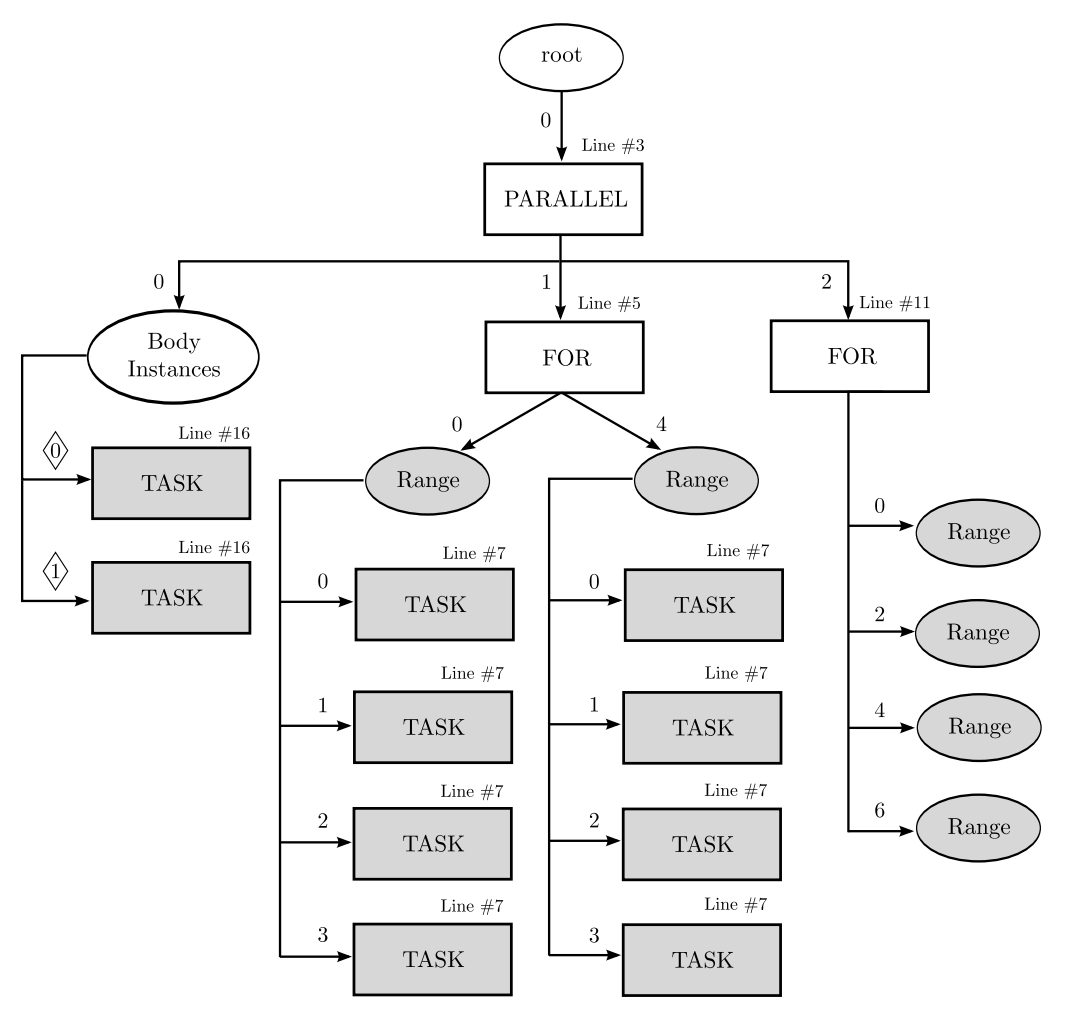

Fig. 2. OpenMP Label Graph of example program, where gray highlights the profiled XUs. Memory addresses are replaced by line numbers for purposes of illustration.

'work' required to transform the bins of $p$ such that they are equivalent to the bins of $q$, where the bins in each histogram contain an amount of earth specified by its weight. Work between two bins $i$ and $j$ is defined as $W_{i j}=\omega_{i j} \cdot \operatorname{dist}\left(\lambda_{i}, \lambda_{j}\right)$ with $\omega_{i j}$ representing the weight moved from $i$ to $j$ and dist representing the ground distance between the two bins that is based on the bin locations $\lambda_{i}$ and $\lambda_{j}$ respectively. EMD is then solved as the overall minimum amount of work necessary to either deplete the weight of $p$ or to fulfill the weight requirement of $q$, and is therefore a formulation of the transportation problem, with the bins of $p$ representing producers and the bins of $q$ representing consumers.

Assuming the total weight in $p$ is equal to the total weight in $q$, which is the case in this paper as described below, the EMD is equivalent to the first Mallow's distance and the first Wasserstein Distance in mathematics [7].

\subsection{Execution Profile Dissimilarity}

We now define our usage of EMD, that we term EPD. The similarity of the complete set of hardware event monitoring data within a profile cannot be directly calculated against a single reference profile when its profiled hardware events 
are not simultaneously compatible on the system. Therefore we define a reference profile for each possible pair of the hardware events. EPD is then given as the geometric mean of its calculated EPDs against each reference profile, with respect to each pair of hardware events.

We denote the pair of hardware events in a given reference profile as $E_{\mathrm{ref}}$. The two profiles are each represented as a two-dimensional histogram where each event $e \in E_{\text {ref }}$ forms a dimension. The histograms are created by dividing each event's range of values in the reference profile into $n$ equally spaced intervals, to produce a two-dimensional grid. Each cell of this grid is a histogram bin to which the XUs are assigned based on their associated hardware event counts.

EPD defines a histogram bin's weight as the proportion of XUs allocated to the bin from the profile. The distance function $\operatorname{dist}\left(\lambda_{i}, \lambda_{j}\right)$ is defined as the Euclidean distance between the two bin locations, where a bin location is specified as a two-dimensional Cartesian coordinate. The coordinate component, with respect to the dimension for one of the hardware events $e$, is given by the distance along that dimension from the histogram's origin to the mean event count for $e$ calculated from the XUs populated in the bin. This distance is expressed in fractional bin-intervals, where the bin interval for an event $e$ is equivalent to its value range in the reference divided by $n$.

The EPD between the two histograms is then calculated by solving the EMD algorithm following these definitions. The result is normalized according to the Calibration EPD. We define the Calibration EPD as the mean dissimilarity calculated between the profiles of repeated reference executions of the program, meaning it therefore quantifies the average expected variability of the event counts. The final EPD result between the target evaluation profile and a particular reference profile is then its mean EPD calculated against each of the repeat reference profiles.

\subsection{Experimental Methodology}

We evaluate our combination approach against HEM on a 32-core machine equipped with Intel Xeon E5-2690 processors running with Hyper-Threading enabled and an operating frequency of $2.93 \mathrm{GHz}$, with $396 \mathrm{~GB}$ main memory distributed over two NUMA nodes. The machine was configured with version 3.19 of the Linux kernel, using the kernel's default multiplexing implementation of the perf_event subsystem with the default multiplexing scheduling period of $4 \mathrm{~ms}$ for HEM. In our experiments, we used the FastEMD library [16].

For evaluation, the loop-based parallel benchmarks $M G$ and $C G$ from the $\mathrm{C}$ implementation of the NPB benchmark suite [13,15], and a custom task-parallel OpenMP C implementation of the branch-and-bound solution to the knapsack problem were used. Two versions of $M G$ and $C G$ programs were profiled, one using static loop scheduling and the other using dynamic scheduling. The dynamic scheduling was configured with chunk sizes of 10 and 100 respectively.

For each benchmark, each reference execution was repeated 3 times and the mean dissimilarity between each repeat was calculated as the Calibration EPD for the reference event-pair. The set of profiles to be combined were executed and 


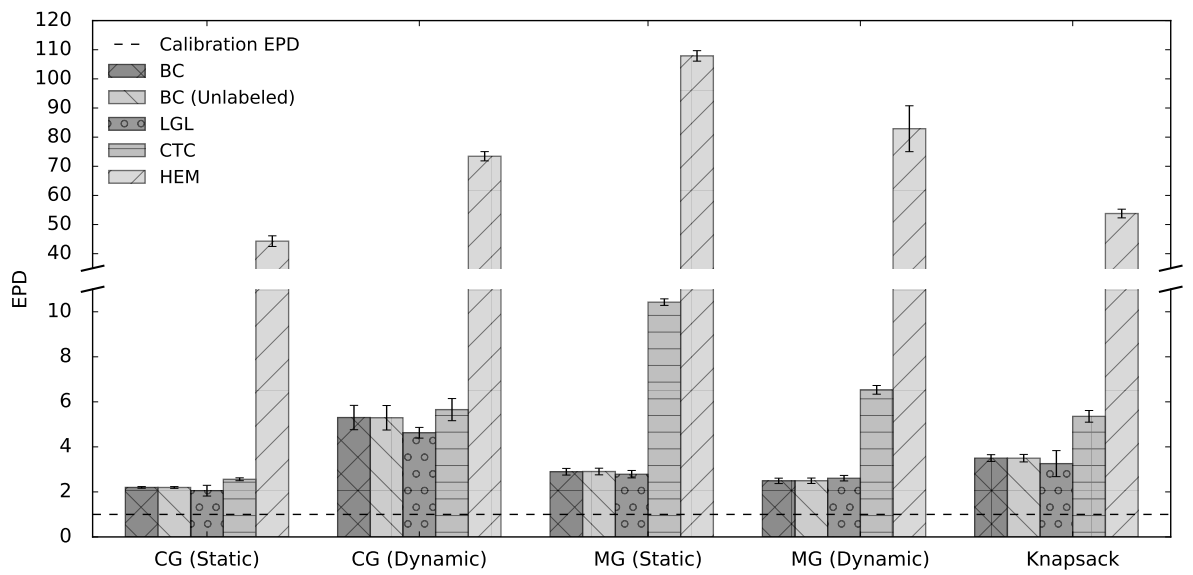

Fig. 3. EPD results for generated hardware event profiles

the combination strategies were applied to produce a set of combined profiles. The EPD of each combined profile against each reference pair was calculated as the mean EPD between the profile and each repeat reference profile corresponding to the pair. The final EPD value for a profile was then the geometric mean of the EPD calculated across the full set of reference pairs. This process was repeated 3 times, with the final EPD result for a strategy calculated as the mean $\mathrm{EPD}$ of the repeat combined profile, and error calculated as standard deviation.

\subsection{Results}

Figure 3 shows the resulting EPDs for the different combination schemes when applied to the 5 combinations of benchmarks and loop schedules. HEM displays by far the highest dissimilarities compared to simultaneous profiling of the events. This major decrease in accuracy is inherent to single-profile sampling techniques like HEM: since it is impossible to monitor all hardware events throughout the entire execution, a significant part of the data is obtained through statistical interpolation. Depending on the number of events, the multiplexing period and the duration of XUs, this might result in event counts for XUs interpolated from the values of previously executed XUs with different characteristics. The large number of hardware events that can be monitored on modern systems and the short durations of XUs in OpenMP programs would require a sampling period that is significantly shorter than the default $4 \mathrm{~ms}$ in the experimental setup in order to obtain samples for all events for each XU. However, a significantly shorter multiplexing period would incur significant overhead and generate biased results. Therefore, HEM provides meaningful results only for a limited set of analysis scenarios.

The multi-execution profile combination strategies achieve hardware event profiles with vastly more accurate results. The EPD values for these are close 
to the Calibration EPD. For most of the benchmarks, CTC clearly produces less accurate combined profiles than the other combination strategies. The CTC strategy assumes that the same XUs perform the same amount of work, execute in the same order and on the same CPUs across the multiple executions of an OpenMP program. The higher EPD results for CTC indicate that this assumption is not valid for most of the benchmarks. However, the results for CTC applied to the statically scheduled $C G$ benchmark are close to the Calibration EPD, meaning that, as expected, the validity of this assumption heavily depends on the nature of the benchmark. Furthermore, as no significant event-scheduling work is required when monitoring the executions necessary for our combination approach, each monitored execution does not exhibit the significant monitoring overhead produced during HEM executions with short mutiplexing periods.

Comparing the results of the LGL and BC combination strategies, there is no clear difference in accuracy, as their resulting EPDs are similarly close to the Calibration EPD across each of the benchmarks. The graph also shows that labeling in $\mathrm{BC}$ only has little influence on the accuracy, as indicated by the similar results for $\mathrm{BC}$ and $\mathrm{BC}$ (Unlabeled). This suggests that the additional trace processing required to apply labels XUs is not necessary to obtain accurate hardware event profiles and that our combination approach may be used directly on OpenMP runtimes that are unable to produce the necessary instrumentation for labeling purposes.

\section{Conclusion}

We presented a new approach for building accurate and complete hardware profiles of OpenMP programs that consists of combining information gathered from multiple executions of the same program into a unique, coherent execution profile. We presented three combination strategies: Chronological Type Combination (CTC), Label Graph Location (LGL) and Behavior Clustering (BC). We introduced CTC as a novel combination strategy technique and presented the work required to adapt LGL and BC to OpenMP, mainly consisting in an appropriate labeling scheme for OpenMP execution units and constructs. based on our EPD metric, a variant of the Earth Mover's Distance, we showed that our approach can be used to build execution profiles that contain information about all hardware event types available, while achieving significantly better accuracy than Hardware Event Multiplexing.

\section{References}

1. L. Adhianto, S. Banerjee, M. Fagan, M. Krentel, G. Marin, J. Mellor-Crummey, and N. R. Tallent. HPCTOOLKIT: Tools for Performance Analysis of Optimized Parallel Programs. Concurrency and Computation: Practice $E$ Experience, 22(6):685701, April 2010.

2. Intel Corporation. Intel VTune Amplifier, 2017. https://software.intel.com/enus/intel-vtune-amplifier-xe, accessed 30/04/2017. 
3. M. Dimakopoulou, S. Eranian, N. Koziris, and N. Bambos. Reliable and efficient performance monitoring in Linux. Proceedings of the International Conference for High Performance Computing, Networking, Storage and Analysis, page 34, 2016.

4. A. Drebes, J.-B. Bréjon, A. Pop, K. Heydemann, and A. Cohen. Language-Centric Performance Analysis of OpenMP Programs with Aftermath. OpenMP: Memory, Devices, and Tasks: 12th International Workshop on OpenMP, IWOMP 2016, Nara, Japan, October 5-7, 2016, Proceedings, pages 237-250, 2016.

5. A. Drebes, A. Pop, K. Heydemann, A. Cohen, and N. Drach-Temam. Aftermath: A graphical tool for performance analysis and debugging of fine-grained task-parallel programs and run-time systems. In 7th Workshop on Programmability Issues for Heterogeneous Multicores (MULTIPROG), Vienna, Austria, 2014.

6. M. Hauswirth, A. Diwan, P. F. Sweeney, and M. C. Mozer. Automating vertical profiling. In Proceedings of the 20th Annual ACM SIGPLAN Conference on Objectoriented Programming, Systems, Languages, and Applications, OOPSLA '05, pages 281-296, New York, NY, USA, 2005. ACM.

7. E. Levina and P. Bickel. The Earth Mover's distance is the Mallows distance: some insights from statistics. In Proceedings Eighth IEEE International Conference on Computer Vision. ICCV 2001, volume 2, pages 251-256 vol.2, 2001.

8. R. V. Lim, D. Carrillo-Cisneros, and I. D. Scherson. Computationally Efficient Multiplexing of Events on Hardware Counters. Linux Symposium, pages 101-110, 2014 .

9. W. Mathur and J. Cook. Towards accurate performance evaluation using hardware counters. ITEA Modeling and Simulation Workshop, 2003.

10. W. Mathur and J. Cook. Improved estimation for software multiplexing of performance counters. In Proceedings - IEEE Computer Society's Annual International Symposium on Modeling, Analysis, and Simulation of Computer and Telecommunications Systems, MASCOTS, volume 2005, pages 23-32. IEEE, 2005.

11. A. Muddukrishna, P. A. Jonsson, and M. Brorsson. Characterizing task-based OpenMP programs. PloS ONE, 10(4):e0123545, 2015.

12. T. Mytkowicz, P. F. Sweeney, M. Hauswirth, and A. Diwan. Time Interpolation: So Many Metrics, So Few Registers. In 40th Annual IEEE/ACM International Symposium on Microarchitecture (MICRO 2007), pages 286-300. IEEE, 2007.

13. NASA. NAS Parallel Benchmarks. https://www.nas.nasa.gov/publications/npb.html, accessed 30/04/2017.

14. R. Neill, A. Drebes, and A. Pop. Fuse: Accurate multiplexing of hardware performance counters across executions, 2017.

15. University of Versailles Saint Quentin en Yvelines. NAS Parallel Benchmarks 3.0 Unofficial OpenMP C Version, 2014. https://github.com/benchmarksubsetting/NPB3.0-omp-C, accessed 30/04/2017.

16. O. Pele and M. Werman. A linear time histogram metric for improved sift matching. Computer Vision-ECCV 2008, pages 495-508, 2008.

17. A. Pop and A. Cohen. OpenStream: Expressiveness and data-flow compilation of OpenMP streaming programs. ACM Transactions on Architecture and Code Optimization, 9(4):53:1-53:25, jan 2013.

18. Y. Rubner, C. Tomasi, and L. J. Guibas. The Earth Mover's Distance as a Metric for Image Retrieval. International Journal of Computer Vision, 40(2):99-121, 2000.

19. S. S. Shende and A. D. Malony. The Tau Parallel Performance System. The International Journal of High Performance Computing Applications, 20(2):287311, May 2006. 